J. Math. Phys. 47, 082106-15 (2006)

DMA[IMECC]UNICAMP

\title{
ANALYTIC PLANE WAVE SOLUTIONS FOR THE QUATERNIONIC POTENTIAL STEP
}

\author{
Stefano De Leo ${ }^{1}$, Gisele C. Ducati ${ }^{2}$, and Tiago M. Madureira ${ }^{2}$ \\ 1 Department of Applied Mathematics, University of Campinas \\ PO Box 6065, SP 13083-970, Campinas, Brazil \\ deleo@ime.unicamp.br \\ 2 Department of Mathematics, University of Parana \\ PO Box 19081, PR 81531-970, Curitiba, Brazil \\ ducati@mat.ufpr.br \\ tmadureira@mat.ufpr.br
}

Submitted: April, 2006. Revised: May, 2006.

\begin{abstract}
By using the recent mathematical tools developed in quaternionic differential operator theory, we solve the Schrödinger equation in presence of a quaternionic step potential. The analytic solution for the stationary states allows to explicitly show the qualitative and quantitative differences between this quaternionic quantum dynamical system and its complex counterpart. A brief discussion on reflected and transmitted times, performed by using the stationary phase method, and its implication on the experimental evidence for deviations of standard quantum mechanics is also presented. The analytic solution given in this paper represents a fundamental mathematical tool to find an analytic approximation to the quaternionic barrier problem (up to now solved by numerical method).
\end{abstract}

PACS. 02.30. $\mathrm{Tb}-03.65 . \mathrm{Ca}$

\section{INTRODUCTION}

Since a quaternionic equation can be equivalently written as a two-component complex equation, it is natural to ask whether the quaternionic Schrödinger equation is simply another way of rewriting complex quantum mechanics. The answer to this question is given in his famous book [1] by Adler. Probabilities in quaternionic dynamical system are different from those of standard complex theory. In the first papers on the quaternionic Schrödinger equation deviations from complex quantum mechanics were studied by considering quaternionic perturbation potentials [2,3]. Recent progresses on the solution of quaternionic differential equations [4-6] have improved the physical discussion on quaternionic tunnelling phenomena [7] and bound states [8].

In this paper, an interesting simple quaternionic quantum mechanical system is analytically solved. This allows to discuss both qualitative and quantitative differences between quaternionic and complex quantum mechanics. The explicit stationary wave solution for the quaternionic potential step shows some important results which could be very useful in looking for deviations from the standard quantum theory. For example, the quaternionic step diffusion is characterized by reflected and transmitted waves which are not instantaneous. The analytic solution is also very useful to understand the effect that quaternionic potentials play on the phase of stationary waves. The advantage to analytically solve a quaternionic problem is surely represented by the possibility to deeply study the quaternionic solution and understand where and if deviations from complex quantum theory could be seen. 


\section{QUATERNIONIC SCHRÖDINGER EQUATION}

In the quaternionic formulation of non-relativistic quantum mechanics, the dynamics of a particle without spin subject to the influence of the anti-hermitian scalar potential,

$$
i V_{1}(\boldsymbol{r}, t)+j V_{2}(\boldsymbol{r}, t)+k V_{3}(\boldsymbol{r}, t),
$$

is described by

$$
\hbar \partial_{t} \Psi(\boldsymbol{r}, t)=\left[i \frac{\hbar^{2}}{2 m} \nabla^{2}-i V_{1}(\boldsymbol{r}, t)-j V_{2}(\boldsymbol{r}, t)-k V_{3}(\boldsymbol{r}, t)\right] \Psi(\boldsymbol{r}, t)
$$

with

$$
V_{1,2,3}:\left(\mathbb{R}^{3}, \mathbb{R}\right) \rightarrow \mathbb{R} \quad \text { and } \quad \Psi:\left(\mathbb{R}^{3}, \mathbb{R}\right) \rightarrow \mathbb{H}
$$

Eq.(1) is known as the Schrödinger equation for quaternionic quantum mechanics [1]. It is natural to try to relate the new results coming from this quaternionic formulation with the well know phenomena discussed in the standard textbooks of (complex) quantum mechanics [9-11]. In this spirit, the complex limit, i.e. $V_{2,3} \rightarrow 0$, surely represents a useful mathematical tool to test quaternionic calculations and to understand, by explicitly showing the difference between the quaternionic and complex formulation, if and where quaternionic deviations from standard quantum mechanics could be seen and investigated.

The linearity in $\partial_{t}$ of the evolution time operator in Eq.(1) guarantees to obtain a positive probability density

$$
\rho(\boldsymbol{r}, t)=\bar{\Psi}(\boldsymbol{r}, t) \Psi(\boldsymbol{r}, t)
$$

together with a continuity equation

$$
\partial_{t} \rho(\boldsymbol{r}, t)+\nabla \cdot \boldsymbol{J}(\boldsymbol{r}, t)=0
$$

To find the explicit form of the current density $\boldsymbol{J}(\boldsymbol{r}, t)$, let us first derive the Schrödinger equation for $\bar{\Psi}(\boldsymbol{r}, t)$ [the quaternionic conjugate of $\Psi(\boldsymbol{r}, t)$, i.e. $(i, j, k) \rightarrow-(i, j, k)]$,

$$
\hbar \partial_{t} \bar{\Psi}(\boldsymbol{r}, t)=-\frac{\hbar^{2}}{2 m} \nabla^{2} \bar{\Psi}(\boldsymbol{r}, t) i+\bar{\Psi}(\boldsymbol{r}, t)\left[i V_{1}(\boldsymbol{r}, t)+j V_{2}(\boldsymbol{r}, t)+k V_{3}(\boldsymbol{r}, t)\right]
$$

Combining Eq.(1) [multiplied from the left by $\bar{\Psi}(\boldsymbol{r}, t)$ ] and Eq.(4) [multiplied from the right by $\Psi(\boldsymbol{r}, t)$ ], we obtain

$$
\partial_{t}[\bar{\Psi}(\boldsymbol{r}, t) \Psi(\boldsymbol{r}, t)]+\frac{\hbar}{2 m}\left\{\left[\nabla^{2} \bar{\Psi}(\boldsymbol{r}, t)\right] i \Psi(\boldsymbol{r}, t)-\bar{\Psi}(\boldsymbol{r}, t) i \nabla^{2} \Psi(\boldsymbol{r}, t)\right\}=0
$$

Consequently, the density current in quaternionic quantum mechanics is formally equal to that one of the usual complex theory, i.e.

$$
\boldsymbol{J}(\boldsymbol{r}, t)=\frac{\hbar}{2 m}\{[\nabla \bar{\Psi}(\boldsymbol{r}, t)] i \Psi(\boldsymbol{r}, t)-\bar{\Psi}(\boldsymbol{r}, t) i \nabla \Psi(\boldsymbol{r}, t)\} .
$$

It is worth pointing out that, due to the non commutativity nature of quaternions, the position of the imaginary unit $i$ is not a choice but it is imposed by the anti-hermiticity of evolution time operator in Eq.(1).

\section{- TIME INDEPENDENT POTENTIALS}

In this paper, we are going to be concerned with a particle in a time independent potential. In complex quantum mechanics, the rapid spatial variations of a square potential introduce purely quantum effects in the motion of the particle. The same is valid for perturbative quaternionic potentials. Before beginning our investigation, we shall discuss some important mathematical properties of the quaternionic Schrödinger equation in the presence of time independent potentials,

$$
\hbar \partial_{t} \Psi(\boldsymbol{r}, t)=\left[i \frac{\hbar^{2}}{2 m} \nabla^{2}-i V_{1}(\boldsymbol{r})-j V_{2}(\boldsymbol{r})-k V_{3}(\boldsymbol{r})\right] \Psi(\boldsymbol{r}, t) .
$$


Taking into account that $\Psi(\boldsymbol{r}, t)$ is a quaternionic function, we apply the method of separation of variables with the time dependent function appearing in the right hand side [4],

$$
\Psi(\boldsymbol{r}, t)=\Phi(\boldsymbol{r}) \exp \left[-\frac{i}{\hbar} E t\right],
$$

with

$$
\Phi: \mathbb{R}^{3} \rightarrow \mathbb{H} .
$$

This stationary solution of the Schrödinger equation leads to a time-independent probability density $\rho(\boldsymbol{r})$. Consequently, the current density satisfies

$$
\boldsymbol{\nabla} \cdot\{[\nabla \bar{\Phi}(\boldsymbol{r})] i \Phi(\boldsymbol{r})-\bar{\Phi}(\boldsymbol{r}) i \nabla \Phi(\boldsymbol{r})\}=0 .
$$

By using the separation of variable (7), Eq.(6) reduces to the following quaternionic (right) eigenvalue problem $[12,13]$,

$$
\left[i \frac{\hbar^{2}}{2 m} \nabla^{2}-i V_{1}(\boldsymbol{r})-j V_{2}(\boldsymbol{r})-k V_{3}(\boldsymbol{r})\right] \Phi(\boldsymbol{r})+\Phi(\boldsymbol{r}) i E=0 .
$$

\section{- TIME REVERSAL INVARIANCE}

From Eq. (6), we can immediately obtain the time-reversed Schrödinger equation

$$
\hbar \partial_{t} \Psi_{T}(\boldsymbol{r},-t)=-\left[i \frac{\hbar^{2}}{2 m} \nabla^{2}-i V_{1}(\boldsymbol{r})-j V_{2}(\boldsymbol{r})-k V_{3}(\boldsymbol{r})\right] \Psi_{T}(\boldsymbol{r},-t) .
$$

In complex quantum mechanics the $*$-conjugation yields a time-reversed version of the original Schrödinger equation. In quaternionic quantum mechanics there does not exist a universal time reversal operator [1]. Only a restricted class of time-independent quaternionic potentials $\left[V_{2}(\boldsymbol{r}) \propto V_{3}(\boldsymbol{r})\right]$, i.e.

$$
W(\boldsymbol{r})=V_{2}(\boldsymbol{r})-i V_{3}(\boldsymbol{r})=|W(\boldsymbol{r})| \exp [i \theta] \quad(\theta=\text { const }),
$$

is time reversal invariant. For these potentials,

$$
\Psi_{T}(\boldsymbol{r},-t)=u \Psi(\boldsymbol{r}, t) \bar{u}, \quad u=k \exp [i \theta] .
$$

In the standard quantum mechanics limit, due to the complex nature of the wave function $\Psi_{\mathrm{C}}(\boldsymbol{r}, t)$, we recover the well known result

$$
\Psi_{\mathrm{C}, T}(\boldsymbol{r},-t)=\Psi_{\mathbb{C}}^{*}(\boldsymbol{r}, t) .
$$

\section{- ONE-DIMENSIONAL SQUARE POTENTIALS}

Let us consider one-dimensional potentials. In the case of square shapes, the potential is a quaternionic constant in certain regions of space. In such regions, the stationary wave function $\Phi(x)$ is obtained by solving the following second order differential equation with (left) constant quaternionic coefficients,

$$
\left[i \frac{\hbar^{2}}{2 m} \Phi^{\prime \prime}(x)-i V_{1}-j V_{2}-k V_{3}\right] \Phi(x)=-\Phi(x) i E .
$$

It is not our purpose here to discuss the theory of quaternionic differential equations and we refer the interested reader to the papers cited in refs. [4-6] where a detailed exposition of the subject is found. The solution of Eq.(12) is

$$
\Phi(x)=(1+j w)\left\{\exp \left[\nu_{-} x\right] c_{1}+\exp \left[-\nu_{-} x\right] c_{2}\right\}+(z+j)\left\{\exp \left[\nu_{+} x\right] c_{3}+\exp \left[-\nu_{+} x\right] c_{4}\right\},
$$

where $c_{1, \ldots, 4}$ are complex coefficients to be determined by the boundary conditions and

$$
\begin{aligned}
\nu_{ \pm} & =\sqrt{2 m\left(V_{1} \pm \sqrt{E^{2}-V_{2}^{2}-V_{3}^{2}}\right)} / \hbar, \\
z & =i\left(V_{2}+i V_{3}\right) /\left(E+\sqrt{E^{2}-V_{2}^{2}-V_{3}^{2}}\right), \\
w & =-i\left(V_{2}-i V_{3}\right) /\left(E+\sqrt{E^{2}-V_{2}^{2}-V_{3}^{2}}\right) \quad \in \mathbb{C}(1, i) .
\end{aligned}
$$


In the free potential region $\left(V_{1,2,3}=0\right)$ the previous solution reduces to

$$
\Phi(x)=\exp [i \epsilon x] c_{1}+\exp [-i \epsilon x] c_{2}+j\left\{\exp [\epsilon x] c_{3}+\exp [-\epsilon x] c_{4}\right\},
$$

where

$$
\epsilon=\sqrt{2 m E} / \hbar \in \mathbb{R}
$$

\section{BOUNDED SOLUTIONS AND CURRENT DENSITY}

Let us now calculate the stationary states in the case of a quaternionic step potential. The procedure follows the standard one. We use Eq.(13) in the region where the potential is a constant and Eq.(14) in the free region. We then impose that such solutions remain bounded and, finally, we match these functions by requiring the continuity of $\Phi(x)$ and its derivative in $x=0$. Before proceeding with our calculations, the only point deserving further discussion concerns the classification of the energy zones in the potential region (in order to distinguish between partial and total reflection). To do this, we have to analyze the complex exponential factors $\nu_{ \pm}$. The possible cases are sketched in the following figure

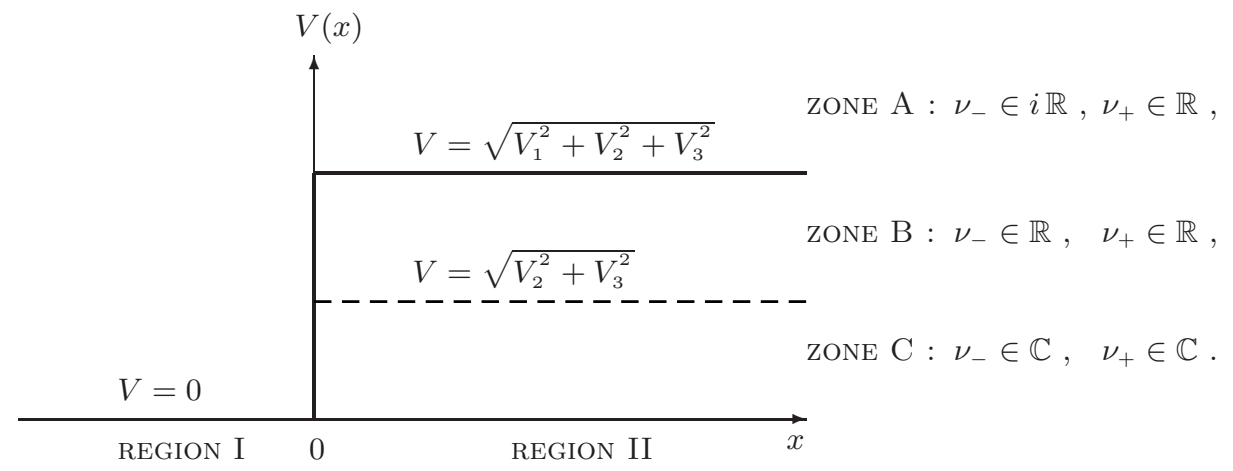

To avoid any confusion between real and imaginary coefficients and to facilitate the reading of this paper, in the sequel, we shall adopt the following notation

$$
\begin{array}{lll}
\text { ZONE A : } & \nu_{-}=i \rho_{-}, & \nu_{+}, \\
\text {ZONE B : } & \nu_{-}, & \nu_{+}, \\
\text {ZONE C : } & \nu_{-}=\sigma_{+}-i \sigma_{-}, & \nu_{+}=\sigma_{+}+i \sigma_{-},
\end{array}
$$

where

$$
\rho_{-}=\sqrt{\frac{2 m}{\hbar^{2}}\left(\sqrt{E^{2}-V_{2}^{2}-V_{3}^{2}}-V_{1}\right)}, \sigma_{ \pm}=\sqrt{\frac{m}{\hbar^{2}}\left(\sqrt{V_{1}^{2}+V_{2}^{2}+V_{3}^{2}-E^{2}} \pm V_{1}\right)} \in \mathbb{R} .
$$

\section{- REGION I}

For the solution to remain bounded when $x \rightarrow-\infty$, it is necessary to have $c_{4}=0$ in Eq.(14). So, the solution in region I becomes

$$
\Phi_{\mathrm{I}}(x)=e^{i \epsilon x}+r e^{-i \epsilon x}+j \tilde{r} e^{\epsilon x},
$$

where $c_{2}=r$ and $c_{3}=\tilde{r}$ represent the reflection coefficients to be determined by the matching conditions. From Eq.(8), we immediately find the constant value of the current density in this region, i.e.

$$
J_{\mathrm{I}}=\left(1-|r|^{2}\right) \hbar \epsilon / m \text {. }
$$




\section{- REGION II - ZONE A: PARTIAL REFLECTION}

The condition on the boundedness of the solution implies that $c_{3}=0$ in Eq.(13). Since, the incident particle is coming from $x=-\infty$, we also have to impose $c_{2}=0$. The stationary wave function, in zone $\mathrm{A}$, is then given by

$$
\Phi_{\mathrm{II}, A}(x)=(1+j w) t e^{i \rho_{-} x}+(z+j) \tilde{t} e^{-\rho_{+} x},
$$

where $c_{1}=t$ and $c_{4}=\tilde{t}$ represent the transmission coefficients to be determined by the matching conditions. In this region the current density is

$$
J_{\mathrm{II}, A}=\left(1-|w|^{2}\right)|t|^{2} \hbar \rho_{-} / m .
$$

This means a non-null transmission probability and consequently partial reflection in region I.

\section{- REGION II - ZONE B: TOTAL REFLECTION}

For the solution to remain bounded when $x \rightarrow+\infty$, it is necessary that $c_{2}=c_{4}=0$ in Eq.(13). Thus, the solution in zone B is

$$
\Phi_{\mathrm{II}, B}(x)=(1+j w) t e^{-\nu_{-} x}+(z+j) \tilde{t} e^{-\nu_{+} x} .
$$

In this zone, the current density is null

$$
J_{\mathrm{II}, B}=0 .
$$

This characterizes a total reflection in region I.

\section{- REGION II - ZONE C: TOTAL REFLECTION}

The boundedness condition of the solution implies that $c_{1}=c_{3}=0$ in Eq.(13). Thus, we have

$$
\Phi_{\mathrm{II}, C}(x)=\left[(1+j w) t e^{i \sigma_{-} x}+(z+j) \tilde{t} e^{-i \sigma_{-} x}\right] e^{-\sigma_{+} x},
$$

with

$$
w=-i \frac{V_{2}-i V_{3}}{\sqrt{V_{2}^{2}+V_{3}^{2}}} e^{-i \varphi}, \quad z=i \frac{V_{2}+i V_{3}}{\sqrt{V_{2}^{2}+V_{3}^{2}}} e^{-i \varphi} \quad \text { and } \quad \varphi=\arctan \left[\frac{\sqrt{V_{2}^{2}+V_{3}^{2}-E^{2}}}{E}\right] .
$$

As in the previous zone, the current density is null

$$
J_{\mathrm{II}, C}=0 .
$$

This implies total reflection in region I.

\section{- RELATION BETWEEN REFLECTION AND TRANSMISSION COEFFICIENTS}

The stationary wave solution of the Schrödinger equation in the presence of a quaternionic step potential can be then expressed in terms of complex reflection $(r, \tilde{r})$ and transmission $(t, \tilde{t})$ coefficients:

$$
\begin{aligned}
& \Phi_{\mathrm{I}}(x)=e^{i \epsilon x}+r e^{-i \epsilon x}+j \tilde{r} e^{\epsilon x}, \\
& \Phi_{\mathrm{II}}(x)= \begin{cases}(1+j w) t e^{i \rho_{-} x}+(z+j) \tilde{t} e^{-\nu_{+} x} & \text { zONE A }, \\
(1+j w) t e^{-\nu_{-} x}+(z+j) \tilde{t} e^{-\nu_{+} x} & \text { zONE B }, \\
{\left[(1+j w) t e^{i \sigma_{-} x}+(z+j) \tilde{t} e^{-i \sigma_{-} x}\right] e^{-\sigma_{+} x}} & \text { zONE C } .\end{cases}
\end{aligned}
$$


As we saw, the current density assumes a constant value. This value has been calculated in the free potential region and in each of the three different zones of region II. The continuity of $\Phi(x)$ and its derivative in $x=0$ implies the continuity of the current density, i.e. $J_{\mathrm{I}}=J_{\mathrm{II}}$. This gives an immediate relation between reflection and transmission coefficients,

$$
R+T=1
$$

with

$$
\begin{array}{ll}
R=|r|^{2} \text { and } T=\frac{\rho_{-}}{\epsilon}\left(1-|w|^{2}\right)|t|^{2} & \text { for } E>\sqrt{V_{1}^{2}+V_{2}^{2}+V_{3}^{2}}, \\
R=|r|^{2} \text { and } T=0 & \text { for } E<\sqrt{V_{1}^{2}+V_{2}^{2}+V_{3}^{2}}
\end{array} .
$$

Observe that, both in complex and quaternionic quantum mechanics, to find the relation between $R$ and $T$ we do not have the necessity to find the explicit value of plane wave coefficients $r$ and $t$.

\section{EXPLICIT PLANE WAVE SOLUTIONS}

The usual method for determining the stationary states in a square potential requires the continuity of $\Phi(x)$ and its derivative at the point where the potential is discontinuous (in this case $x=0$ ). Then, we impose that

$$
\begin{aligned}
& \Phi_{\mathrm{I}}(0)=\Phi_{\mathrm{II}}(0) \\
& \Phi_{\mathrm{I}}^{\prime}(0)=\Phi_{\mathrm{II}}^{\prime}(0) .
\end{aligned}
$$

\section{- REGION II - ZONE A: CONTINUITY}

Matching the conditions at $x=0$, we get

$$
\begin{aligned}
1+r+j \tilde{r} & =(1+j w) t+(z+j) \tilde{t}, \\
i \epsilon(1-r)+j \epsilon \tilde{r} & =(1+j w) i \rho_{-} t-(z+j) \nu_{+} \tilde{t} .
\end{aligned}
$$

After separating the complex from the pure quaternionic part, we find

$$
\begin{aligned}
& 1+r=t+z \tilde{t}, \\
& \tilde{r}=w t+\tilde{t}, \\
& 1-r=\frac{\rho_{-}}{\epsilon} t+i \frac{\nu_{+}}{\epsilon} z \tilde{t}, \\
& \tilde{r}=i \frac{\rho_{-}}{\epsilon} w t-\frac{\nu_{+}}{\epsilon} \tilde{t},
\end{aligned}
$$

which gives

$$
\begin{aligned}
t & =\frac{2 \epsilon}{\epsilon+\rho_{-}}\left[1-z w \frac{\epsilon+i \nu_{+}}{\epsilon+\nu_{+}} \frac{\epsilon-i \rho_{-}}{\epsilon+\rho_{-}}\right]^{-1}, \\
r & =\frac{\epsilon-\rho_{-}}{2 \epsilon}\left[1-z w \frac{\epsilon-i \nu_{+}}{\epsilon+\nu_{+}} \frac{\epsilon-i \rho_{-}}{\epsilon-\rho_{-}}\right] t, \\
\tilde{t} & =-\frac{\epsilon-i \rho_{-}}{\epsilon+\nu_{+}} w t \\
\tilde{r} & =\frac{\nu_{+}+i \rho_{-}}{\epsilon+\nu_{+}} w t .
\end{aligned}
$$

We have determined the stationary states of a particle in the presence of a quaternionic step potential for plane waves of energy $E>V_{0}=\sqrt{V_{1}^{2}+V_{2}^{2}+V_{3}^{2}}$. In Figure 1, we plot, for different ratios of the complex and pure quaternionic potential $\left(V_{1} / \sqrt{V_{2}^{2}+V_{3}^{2}}\right)$, the four real component of $\Phi(x)$ versus the adimensional space variable $\sqrt{2 m V_{0}} x / \hbar$. In region I, only the complex part of $\Phi(x)$ presents an oscillatory behavior. The pure quaternionic part decreases exponentially due to the presence of 
the evanescent wave $e^{\epsilon x}$. In region II, we have a new oscillatory pure quaternionic wave. It is also important to note here that increasing the value of pure quaternionic potential, we smooth the phase changes expected in the potential region by standard quantum mechanics. Thus, we can conclude that this change in the phase is caused by the complex part of the quaternionic potential.

These plane waves do not represent a physical state for a localized incoming particle. They have to be linearly superposed to form wave packets. It is not our purpose to introduce the wave packet treatment for quaternionic wave functions in this paper. This topic deserves a deeper analysis and is, currently, under investigation. Nevertheless, a simple discussion can done at this stage. By using the stationary phase method [9], we can follow the maximum of the reflected and transmitted wave packets. The use of a real modulation function $g(\epsilon)$ implies that the incident wave packets reaches the point $x=0$ at $t=0$. Any phase in the reflected and/or transmitted waves will introduce a shift in time. To clarify this point, it can be useful to rewrite the reflection and transmission coefficients in temrs of their modulus and phases. By simple algebraic manipulations, we find

$$
\begin{aligned}
r & =\frac{\left(\epsilon-\rho_{-}\right)\left(\epsilon+\nu_{+}\right)-z w\left(\epsilon^{2}-\rho_{-} \nu_{+}\right)+i z w \epsilon\left(\rho_{-}+\nu_{+}\right)}{\left(\epsilon+\rho_{-}\right)\left(\epsilon+\nu_{+}\right)-z w\left(\epsilon^{2}+\rho_{-} \nu_{+}\right)+i z w \epsilon\left(\rho_{-}-\nu_{+}\right)} \\
& =\sqrt{\frac{\left[\left(\epsilon-\rho_{-}\right)\left(\epsilon+\nu_{+}\right)-z w\left(\epsilon^{2}-\rho_{-} \nu_{+}\right)\right]^{2}+z^{2} w^{2} \epsilon^{2}\left(\rho_{-}+\nu_{+}\right)^{2}}{\left[\left(\epsilon+\rho_{-}\right)\left(\epsilon+\nu_{+}\right)-z w\left(\epsilon^{2}+\rho_{-} \nu_{+}\right)\right]^{2}+z^{2} w^{2} \epsilon^{2}\left(\rho_{-}-\nu_{+}\right)^{2}}} \exp \left[i\left(\theta_{n}-\theta_{d}\right)\right]
\end{aligned}
$$

and

$$
\begin{aligned}
t & =\frac{2 \epsilon}{\epsilon+\rho_{-}}\left[\frac{\left(\epsilon+\rho_{-}\right)\left(\epsilon+\nu_{+}\right)}{\left(\epsilon+\rho_{-}\right)\left(\epsilon+\nu_{+}\right)-z w\left(\epsilon-i \rho_{-}\right)\left(\epsilon+i \nu_{+}\right)}\right] \\
& =\frac{2 \epsilon\left(\epsilon+\nu_{+}\right)}{\sqrt{\left[\left(\epsilon+\rho_{-}\right)\left(\epsilon+\nu_{+}\right)-z w\left(\epsilon^{2}-\rho_{-} \nu_{+}\right)\right]^{2}+z^{2} w^{2} \epsilon^{2}\left(\rho_{-}+\nu_{+}\right)^{2}}} \exp \left[-i \theta_{d}\right]
\end{aligned}
$$

where

$$
\begin{aligned}
& \theta_{n}=\arctan \left[\frac{z w \epsilon\left(\rho_{-}+\nu_{+}\right)}{\left(\epsilon-\rho_{-}\right)\left(\epsilon+\nu_{+}\right)-z w\left(\epsilon^{2}-\rho_{-} \nu_{+}\right)}\right], \\
& \theta_{d}=\arctan \left[\frac{z w \epsilon\left(\rho_{-}-\nu_{+}\right)}{\left(\epsilon+\rho_{-}\right)\left(\epsilon+\nu_{+}\right)-z w\left(\epsilon^{2}+\rho_{-} \nu_{+}\right)}\right] .
\end{aligned}
$$

The phases of the reflected and transmitted waves are then given by

$$
\begin{aligned}
& \theta_{r}(\epsilon ; x, t)=\theta_{n}(\epsilon)-\theta_{d}(\epsilon)-\epsilon x-\frac{\hbar \epsilon^{2}}{2 m} t, \\
& \theta_{t}(\epsilon ; x, t)=-\theta_{d}(\epsilon)+\rho_{-}(\epsilon) x-\frac{\hbar \epsilon^{2}}{2 m} t .
\end{aligned}
$$

The stationary phase method suggests that the maximum of the reflected and transmitted waves is found at the point $x=0$ for the following time values:

$$
\begin{aligned}
\tau_{r} & =\frac{m}{\hbar} \frac{\theta_{n}^{\prime}\left(\epsilon_{0}\right)-\theta_{d}^{\prime}\left(\epsilon_{0}\right)}{\epsilon_{0}}, \\
\tau_{t} & =-\frac{m}{\hbar} \frac{\theta_{d}^{\prime}\left(\epsilon_{0}\right)}{\epsilon_{0}},
\end{aligned}
$$

where $\epsilon_{0}$ is the maximum of the modulation function $g(\epsilon)$. In this energy zone $\left(E>V_{0}\right)$, an immediate qualitative difference between complex and quaternionic quantum mechanics is found. For quaternionic potential the reflection and transmission are not instantaneous. We shall come back to this point later.

\section{- REGION II - ZONE A: COMPLEX LIMIT}

Performing the complex limit, $V_{2,3} \rightarrow 0$, we obtain

$$
\begin{aligned}
\nu_{+} & \rightarrow \sqrt{2 m\left(E+V_{1}\right)} / \hbar, \\
\rho_{-} & \rightarrow \sqrt{2 m\left(E-V_{1}\right)} / \hbar, \\
z, w & \rightarrow 0 .
\end{aligned}
$$


From Eq.(27), we find the reflection and transmission coefficient of standard (complex) quantum mechanics

$$
\begin{aligned}
t_{\mathbb{C}} & =2 \sqrt{E} /\left(\sqrt{E}+\sqrt{E-V_{1}}\right), \\
r_{\mathbb{C}} & =\left(\sqrt{E}-\sqrt{E-V_{1}}\right) /\left(\sqrt{E}+\sqrt{E-V_{1}}\right), \\
\tilde{r}_{\mathbb{C}}, \tilde{t}_{\mathbb{C}} & =0 .
\end{aligned}
$$

Due to the real nature of $r_{\mathbb{C}}$ and $t_{\mathbb{C}}$, we find instantaneous reflection and transmission. This means that at time zero, the maximum of the incident, reflected and transmitted waves are at $x=0$.

\section{- REGION II - ZONE B: CONTINUITY}

Matching the continuity conditions at $x=0$, we obtain

$$
\begin{aligned}
t & =\frac{2 \epsilon}{\epsilon+i \nu_{-}}\left[1-z w \frac{\epsilon+\nu_{-}}{\epsilon+i \nu_{-}} \frac{\epsilon+i \nu_{+}}{\epsilon+\nu_{+}}\right]^{-1}, \\
r & =\frac{\epsilon-i \nu_{-}}{2 \epsilon}\left[1-z w \frac{\epsilon+\nu_{-}}{\epsilon-i \nu_{-}} \frac{\epsilon-i \nu_{+}}{\epsilon+\nu_{+}}\right] t, \\
\tilde{t} & =-\frac{\epsilon+\nu_{-}}{\epsilon+\nu_{+}} w t \\
\tilde{r} & =\frac{\nu_{+}-\nu_{-}}{\epsilon+\nu_{+}} w t
\end{aligned}
$$

In Figure 2, we plot the four real component of $\Phi(x)$ versus the adimensional space variable $\sqrt{2 m V_{0}} x / \hbar$. Zone B is characterized by $\sqrt{V_{2}^{2}+V_{3}^{2}}<E<V_{0}$. In Figure 2, we have considered the case $E=V_{0} / \sqrt{2}$. Consequently, the behavior of the stationary waves in this zone is given by the plots corresponding to $\sqrt{V_{2}^{2}+V_{3}^{2}} / V_{0}<1 / \sqrt{2}$. In this zone, due to the presence of evanescent exponentials in the transmitted waves, we find a non-zero probability to find the particle in the region of space where $x$ is positive only for short times. The stationary phase method can be applied to the reflected wave. The coefficient $r$ can be rewritten as follows

$$
r=\frac{\epsilon\left[\left(\epsilon+\nu_{+}\right)-z w\left(\epsilon+\nu_{-}\right)\right]+i\left[z w \nu_{+}\left(\epsilon+\nu_{-}\right)-\nu_{-}\left(\epsilon+\nu_{+}\right)\right]}{\epsilon\left[\left(\epsilon+\nu_{+}\right)-z w\left(\epsilon+\nu_{-}\right)\right]-i\left[z w \nu_{+}\left(\epsilon+\nu_{-}\right)-\nu_{-}\left(\epsilon+\nu_{+}\right)\right]}=\exp [2 i \theta]
$$

where

$$
\theta=\arctan \left[\frac{z w \nu_{+}\left(\epsilon+\nu_{-}\right)-\nu_{-}\left(\epsilon+\nu_{+}\right)}{\epsilon\left(\epsilon+\nu_{+}\right)-z w \epsilon\left(\epsilon+\nu_{-}\right)}\right]
$$

The phase of the reflected wave is then given by

$$
\theta_{r}(\epsilon ; x, t)=2 \theta(\epsilon)-\epsilon x-\frac{\hbar \epsilon^{2}}{2 m} t
$$

It is important to observe that here we have a quantitative difference between complex and quaternionic quantum mechanics. Indeed, also for the standard quantum theory the reflection is not instantaneous. The reflect wave reaches $x=0$ at time

$$
\tau_{r}=2 \frac{m}{\hbar} \frac{\theta^{\prime}\left(\epsilon_{0}\right)}{\epsilon_{0}} .
$$




\section{- REGION II - ZONE B: COMPLEX LIMIT}

Performing the complex limit, $V_{2,3} \rightarrow 0$, we obtain

$$
\begin{aligned}
t \rightarrow t_{\mathbb{C}} & =2 \sqrt{E} /\left(\sqrt{E}+i \sqrt{V_{1}-E}\right), \\
r \rightarrow r_{\mathbb{C}} & =\left(\sqrt{E}-i \sqrt{V_{1}-E}\right) /\left(\sqrt{E}+i \sqrt{V_{1}-E}\right), \\
\tilde{r}, \tilde{t} \rightarrow 0 . &
\end{aligned}
$$

The presence of the phase

$$
\theta_{\mathbb{C}}=\arctan \left(-\sqrt{\frac{V_{1}-E}{E}}\right)
$$

in $r_{\mathbb{C}}$ shows that the reflection is not instantaneous. The delay time [9] is

$$
\tau_{r, \mathrm{c}}=2 \frac{m}{\hbar} \frac{\theta_{\mathbb{c}}^{\prime}\left(\epsilon_{0}\right)}{\epsilon_{0}}=\frac{\hbar}{E_{0}} \sqrt{\frac{E_{0}}{V_{1}-E_{0}}} .
$$

\section{- REGION II - ZONE C: CONTINUITY}

The last zone is characterized by $E<\sqrt{V_{2}^{2}+V_{3}^{2}}$. Continuity conditions give

$$
\begin{aligned}
t & =\frac{2 \epsilon}{\epsilon+\sigma_{-}+i \sigma_{+}}\left[1-z w \frac{\epsilon+\sigma_{+}-i \sigma_{-}}{\epsilon+\sigma_{-}+i \sigma_{+}} \frac{\epsilon-\sigma_{-}+i \sigma_{+}}{\epsilon+\sigma_{+}+i \sigma_{-}}\right]^{-1}, \\
r & =\frac{\epsilon-\sigma_{-}-i \sigma_{+}}{2 \epsilon}\left[1-z w \frac{\epsilon+\sigma_{+}-i \sigma_{-}}{\epsilon-\sigma_{-}-i \sigma_{+}} \frac{\epsilon+\sigma_{-}-i \sigma_{+}}{\epsilon+\sigma_{+}+i \sigma_{-}}\right] t, \\
\tilde{t} & =-\frac{\epsilon+\sigma_{+}-i \sigma_{-}}{\epsilon+\sigma_{+}+i \sigma_{-}} w t, \\
\tilde{r} & =\frac{2 i \sigma_{-}}{\epsilon+\sigma_{+}+i \sigma_{-}} w t .
\end{aligned}
$$

The stationary waves corresponding to this case are also shown in Figure 2, see the plots with $\sqrt{V_{2}^{2}+V_{3}^{2}} / V_{0}>1 / \sqrt{2}$. To discuss the time reflection, we rewrite $r$ in terms of its modulus and phase,

$$
\begin{aligned}
r & =\frac{\left(\epsilon-\sigma_{-}-i \sigma_{+}\right)\left(\epsilon+\sigma_{+}+i \sigma_{-}\right) e^{i \varphi}-\left(\epsilon+\sigma_{+}-i \sigma_{-}\right)\left(\epsilon+\sigma_{-}-i \sigma_{+}\right) e^{-i \varphi}}{\left(\epsilon+\sigma_{+}+i \sigma_{-}\right)\left(\epsilon+\sigma_{-}+i \sigma_{+}\right) e^{i \varphi}-\left(\epsilon+\sigma_{+}-i \sigma_{-}\right)\left(\epsilon-\sigma_{-}+i \sigma_{+}\right) e^{-i \varphi}}= \\
& =\exp [2 i \theta]
\end{aligned}
$$

where

$$
\theta=\arctan \left[\frac{\epsilon\left(\epsilon+\sigma_{+}\right) \tan \varphi+\epsilon \sigma_{-}}{\epsilon \sigma_{-}-\left(\epsilon \sigma_{+}+\sigma_{-}^{2}+\sigma_{+}^{2}\right) \tan \varphi}\right]
$$

In this zone, we do not have a complex limit case. It is important to observe that a new phenomenon appears. The oscillatory behavior of the particle in region II is damped due to the presence of the evanescent wave $e^{-\sigma_{+} x}$. Thus, a non-zero probability to find the particle in the potential region only exists for short times.

\section{RELATIONSHIPS BETWEEN COMPLEX AND QUATERNIONIC QUAN- TUM MECHANICS.}

In the last years, the Schrödinger equation in the presence of quaternionic (constant) potentials has been a matter of study and discussion in literature. This is justified in view of a possible understanding 
of the role that a quaternionic quantum theory could play in the real physical world. As remarked by Adler [1] all known physical phenomena appear to be very well described by complex quantum mechanics. Nevertheless, to see if quaternionic quantum mechanics represents a possible way to describe the nature or if it is only an interesting mathematical exercise, we have to use and test this formalism in simple quantum mechanical systems. With respect to previous works regarding potential barrier diffusion $[2,3,7]$ and potential well bound states [8], in this paper, we have preferred to go back in our analysis of non relativistic quaternionic quantum mechanics by studying the potential step.

The study presented in this paper can be seen as an attempt to understand, by starting from an analytic solution of a simple quantum mechanical system, where and if differences between standard quantum mechanics and theoretical solutions obtained by solving the Schrödinger equation in the presence of a quaternionic step perturbation can be observed. The main difficulty in obtaining quaternionic solutions of a physical problem is due to the fact that, in general, the standard mathematical methods of resolution break down. Nevertheless, the recent progress in quaternionic differential theory [4-6] and linear algebra [12,13] give the possibility to use "new" quaternionic mathematical tools. As a direct consequence of this, we have been able to find an analytic solution for the stationary states in the presence of a quaternionic potential step. This means that we have now (for the first time) the possibility of describing in detail qualitative differences between complex and quaternionic quantum mechanics.

\section{- EXPERIMENTAL PROPOSALS IN QUATERNIONIC QUANTUM MECHANICS.}

The earliest experimental proposals to test quaternionic deviations from complex quantum mechanics [14] suggested that the non commutativity of quaternionic phases could be observed in Bragg scattering by crystal made of three different atoms, in neutron interferometry and in meson regeneration. In 1984, the neutron interferometric experiment was realized by Kaiser, George and Werner [15]. The neutron wave function traversing slabs of two dissimilar materials (titanium and aluminum) should experience the non commutativity of the phase shifts when the order in which the barriers are traversed is reversed. The experimental result showed that the phase shifts commute to better than one part in $3 \times 10^{4}$. To explain this null result, Klein postulated [16] that quaternionic potentials act only for some of the fundamental forces and proposed an experiment for testing possible violations of the Schrödinger equation by permuting the order in which nuclear, magnetic and gravitational potentials act on neutrons in an interferometer.

The first theoretical analysis of two quaternionic potential barriers was developed by Davies and McKellar [3]. In their paper, by translating the quaternionic Schrödinger equation into a pair of coupled complex equations and solving the corresponding complex system by numerical methods, Davies and McKellar showed that, notwithstanding the presence of complex instead of quaternionic phases, the predictions of quaternionic quantum mechanics differ from those of the usual theory. In particular, they pointed out that differently from the complex quantum mechanics prediction, where the left and right transmission amplitudes, $t_{L}$ and $t_{R}$, are equal in magnitude and in phase, in the quaternionic quantum mechanics only the magnitudes $\left|t_{L}\right|$ and $\left|t_{R}\right|$ are equal. So, the measurement of a phase shift should be an indicator of quaternionic effects and of space dependent phase potentials. However, this conclusion leads to the embarrassing question of why there was no phase change in the experiment proposed by Peres [14] and realized by Kaiser, George and Werner [15]. To reconcile the theoretical predictions with the experimental observations, Davies and McKellar reiterated the Klein conclusion and suggested to subject the neutron beam to different interactions in permuted order.

In the final chapter of the Adler book [1], we find and intriguing question. Do the Kayser and colleagues experiment, and the elaborations on it proposed by Klein actually test for residual quaternionic effects? According to the non relativistic quaternionic scattering theory developed by Adler [1]

the answer is clearly no. Experiments to detect a phase shift are equivalent to detect time reversal violation, which so far has not been detectable in neutron-optical experiments.

\section{- QUATERNIONIC POTENTIAL STEP, CP VIOLATION AND KAONS SYSTEM.}

Based on the previous considerations, experimental proposals to test quaternionic deviations from standard quantum mechanics should involve $\mathrm{CP}$ violation dynamical systems. A natural candidate to 
a such investigation could be the system of K-mesons $[17,18]$. The quaternionic time reversal violation potential (see Section II)

$$
W(\boldsymbol{r})=|W(\boldsymbol{r})| \exp [i \theta(\boldsymbol{r})]
$$

should be directly responsible for $\mathrm{CP}$ violation effects. The experimental results on $K_{\mathrm{S}, L}$ (a $K_{L}$ meson decays more often to $\pi^{-} e^{+} \bar{\nu}_{e}$ than to $\pi^{+} e^{-} \nu_{e}$ [19]) could be useful to estimate the modulus and phase of the pure quaternionic part of this "effective" potential.

Once determined the magnitude of the quaternionic perturbation, by using the analytic solution obtained in this paper, could be possible (through a stationary phase analysis) to explicitly calculate the reflection and transmission times of a K-meson particle scattered by a complex potential step in the presence of a quaternionic ( $\mathrm{CP}$ violating) perturbation. In the case of a above-potential incident particle the diffusion from a pure complex potential (standard quantum mechanics) happens instantaneously, i.e.

$$
\tau_{r, \mathbb{C}}=\tau_{t, \mathbb{C}}=0 .
$$

The possibility to analytically solve the corresponding quaternionic problem gives us the chance to see an immediate qualitative difference between complex and quaternionic quantum mechanics. The presence of a quaternionic potential surprisingly modifies the reflection and transmission times which are now non zero,

$$
\tau_{r}=\frac{m}{\hbar} \frac{\theta_{n}^{\prime}\left(\epsilon_{0}\right)-\theta_{d}^{\prime}\left(\epsilon_{0}\right)}{\epsilon_{0}} \text { and } \tau_{t}=-\frac{m}{\hbar} \frac{\theta_{d}^{\prime}\left(\epsilon_{0}\right)}{\epsilon_{0}},
$$

where $\epsilon_{0}$ is the maximum of the wave packet modulation function.

With this paper, we would have liked to close the debate on the role that quaternionic potentials could play in quantum mechanics, but more realistically, we simply contribute to the general discussion. Physical interpretations of quaternionic solutions still represent a delicate question and before proposing a detailed experimental test, we think that more mathematical questions should be addressed and deeply investigated.

\section{- COMPLEX AND QUATERNIONIC GEOMETRIES.}

To give a satisfactory probability interpretation, amplitudes of probability must be defined in associative division algebras [1]. Amplitudes of probabilities defined in non-division algebras fail to satisfy the requirement that in the absence of quantum interference effects, probability amplitude superposition should reduce to probability superposition. The associative law of multiplication (which fails for the octonions) is needed to satisfy the completeness formula and to guarantee that the Schrödinger anti-self-adjoint operator leaves invariant the inner product.

At first glance it appears that we cannot formulate quantum theories by using wave functions defined in non-division or non-associative algebras. This is an erroneous conclusion because the constraint concerns the inner product and not the kind of Hilbert space in which we define our wave functions. Amplitudes of probability have to be given in $\mathbb{C}$ or $\mathbb{H}$ (complex or quaternionic geometry) but vectors in the Hilbert space have no limitation. We can formulate a consistent complexified quaternionic [20-24] or octonionic [25,26] quantum mechanics by adopting complex inner products. The use of complex inner product represents a fundamental tool in applying a Clifford algebraic formalism to physics and plays a fundamental role in looking for geometric interpretation of the algebraic structures in relativistic equations and gauge theories [27-31]. The choice of quaternionic inner product seems to be best adapted to investigate deviations from the standard complex theory in quantum mechanics $[32,33]$ and field theory $[34,35]$

\section{- CONCLUSIONS AND OUTLOOKS.}

We conclude this paper by listing the most interesting features of our analysis and future investigations suggested by our results.

(1) An analytic solution for a simple quantum mechanical system (quaternionic potential step) has been given (previous studies on the quaternionic Schrödinger equation have been performed by numerical calculations).

(2) Our plane wave analysis immediately show qualitative differences between complex and quaternionic quantum mechanics (see for example the reflection and transmission times for above-potential 
diffusion and the oscillatory behavior in the new region below the potential).

(3) A plane wave analysis for a quaternionic barrier can be now developed by using an analytic two step approach.

(4) The plane wave results (valid in the physical situation of complete interference) should be revised by introducing a quaternionic wave packet formalism (particle viewpoint). This should confirm and explain the reflection and transmission times obtained by the stationary phase method.

(5) In the quaternionic barrier analysis, we expect qualitative differences between complex and quaternionic quantum dynamical system.

(5.1) For above-potential diffusion, the quaternionic wave packets will be characterized by new reflection and transmission times with respect to the standard (complex) case [36,37].

(5.2) In the tunnelling zone, the quaternionic Hartman effect have to be investigated and confronted with the standard one which predicts (for a long barrier) instantaneous transmission [38-40].

(5.3) In the new below-potential region, a Klein-like phenomenon [41,42] appears and it should be interpreted within a non-relativistic context.

(6) The natural candidate to quaternionic experimental proposals seems to be the system of $K$-mesons. The above suggested investigations should give a more clear idea about the possibility to really perform an experiment involving non-relativistic oscillating particles and $\mathrm{CP}$ violating potential barriers. This probably should close the debate on the use of a quaternionic mathematical formalism in quantum theories.

\section{References}

1. S. L. Adler, Quaternionic quantum mechanics and quantum fields, (New York: Oxford University Press, 1995).

2. A. J. Davies and B. H. McKellar, "Non-relativistic quaternionic quantum mechanics", Phys. Rev. A 40, 4209-4214 (1989).

3. A. J. Davies and B. H. McKellar, "Observability of quaternionic quantum mechanics", Phys. Rev. A 46, 3671-3675 (1992).

4. S, De Leo and G. C. Ducati, "Quaternionic differential operators, J. Math. Phys. 42, 2236-2265 (2001).

5. S, De Leo and G. C. Ducati , "Solving simple quaternionic differential equations, J. Math. Phys. 44, 2224-2233 (2003).

6. S, De Leo and G. C. Ducati , "Real linear quaternionic differential operators, Comp. Math. Appl. 48, 1893-1903 (2004).

7. S. De Leo, G. Ducati and C. Nishi, "Quaternionic potential in non-relativistic quantum mechanics, J. Phys. A 35, 5411-5426 (2002).

8. S. De Leo and G. C. Ducati, "Quaternionic bound states, J. Phys. A 38, 3443-3454 (2005).

9. C. Choen-Tannoudji, B. Diu and F. Lalöe, Quantum mechanics, (New York: John Wiley \& Sons, 1977).

10. P. T. Matthews, Introduction to quantum mechanics, (New York: McGraw-Hill, 1963).

11. E. Merzbacher, Quantum mechanics, (New York: John Wiley \& Sons, 1970).

12. S. De Leo and G. Scolarici, "Right eigenvalue equation in quaternionic quantum mechanics", J. Phys. A 33, 2971-2995 (2000).

13. S. De Leo, G. Scolarici and L. Solombrino, "Quaternionic eigenvalue problem", J. Math. Phys. 43, 58122995 (2002).

14. A. Peres, "Proposed test for complex versus quaternion quantum theory", Phys. Rev. Lett. 42, 683-686 (1979).

15. H. Kaiser, E. A. George and S. A. Werner, "Neutron interferometric search for quaternions in quantum mechanics", Phys. Rev. A 29, 2276-2279 (1984).

16. A. G. Klein, "Schrödinger inviolate: neutron optical searches for violations of quantum mechanics", Physica B 151, 44-49 (1988).

17. C. Itzykson and J.B. Zuber, Quantum Field Theory, McGraw-Hill, Singapore (1985).

18. J.J. Sakurai, Advanced Quantum Mechanics, Addison-Wesley, New York (1987).

19. S. Eidelman et al., "The Review of Particle Physics", Phys. Lett. B 592, 1 (2004)

20. A.W. Conway, "Quaternion treatment of the electron wave equation", Proc. Roy. Soc. A 162, 145154 (1937).

21. A.W. Conway, "Quaternions and quantum mechanics", Acta Pont. Acad. Scien. 12259277 (1948).

22. S. De Leo, "One component Dirac equation", Int. J. Mod. Phys. A 11, 3973-3985 (1996).

23. S. De Leo and W. A. Rodrigues, "Quaternionic electron theory", Int. J. Theor. Phys. 37, 1511-1529 (1998); ibidem 1707-1720 (1998). 
24. S. De Leo, W. A. Rodrigues and J. Vaz, "Complex geometry and Dirac equation", Int. J. Theor. Phys. 37, 2415-2431 (1998).

25. S. De Leo and K. Abdel-Khalek, "Octonionic quantum mechanics and complex geometry", Prog. Theor. Phys. 96, 823-831 (1996); "Octonionic Dirac equation", ibidem 833-845 (1996).

26. S. De Leo and K. Abdel-Khalek, "Octonionic representations of GL(8,R) and GL(4,C)", J. Math. Phys. 38, 582-598 (1997).

27. D. Hestenes, Spacetime algebra (New York: Gordon and Breach, 1966).

28. A. Lasenby, C. Doran, and S. Gull, "A multivector derivative approach to Lagrangian field theory", Found. Phys. 23, 12951327 (1993).

29. S. Gull, C. Doran, and A. Lasenby, "Electron paths, tunneling and diffraction in the spacetime algebra", Found. Phys. 23, 13291356 (1993).

30. S. De Leo, Z. Oziewicz, W.A. Rodrigues, and J. Vaz, "Dirac Hestenes Lagrangian", Int. J. Th. Phys. 38, 23492369 (1999).

31. A. Lasenby and J. Lasenby, "Applications of geometric algebra in physics and links with engineering", 430457, in E.B. Corrochano and G. Sobczyk, Geometric Algebra with Applications in Science and Engineering (Boston: Birkhauser, 2001) .

32. J. Soucek, Quaternion quantummechanics as a true 3+1dimensional theory of tachyons, J. Phys. A 14 (1981) 16291640.

33. J. Soucek, Quaternion quantum mechanics as a description of tachyons and quarks, Czech. J. Phys. B 29 (1979) 315318.

34. S.L. Adler, "Quaternionic quantum field theory", Phys. Rev. Lett. 55, 783786 (1985).

35. S.L. Adler, "Quaternionic quantum field theory", Comm. Math. Phys. 104, 611656 (1986).

36. A. Anderson, "Multiple scattering approach to one-dimensional potential problem", Am. J. Phys. 57, 230-235 (1989).

37. A. Bernardini, S. De Leo and P. Rotelli, "Above barrier potential diffusion", Mod. Phys. Lett. A 19, 2717-2725 (2004).

38. T.E. Hartman, "Tunnelling of a wave packet", J. Appl. Phys. 33, 3427-3432 (1962).

39. A. Pablo, L. Barbero, H.E. Hernández-Figueroa, and E. Recami, "Propagation speed of evanescent modes", Phys. E 62, 8628-8635 (2000).

40. V.S. Olkhovsky, E. Recami, and J, Jakiel, "Unified time analysis of photon and particle tunnelling", Phys. Rep. 398, 133-178 (2004).

41. O. Klein, "Die Reflexion von Elektronrn an einem Potentialsprun nach der relativistichen Dynamik von Dirac", Z. Phys. 53, 157-165 (1929).

42. M. Soffel, B. Müller, and W. Greiner, "Stability and decay of the Dirac vacuum in external gauge fields", Phys. Rep. 85, 51-122 (1982). 


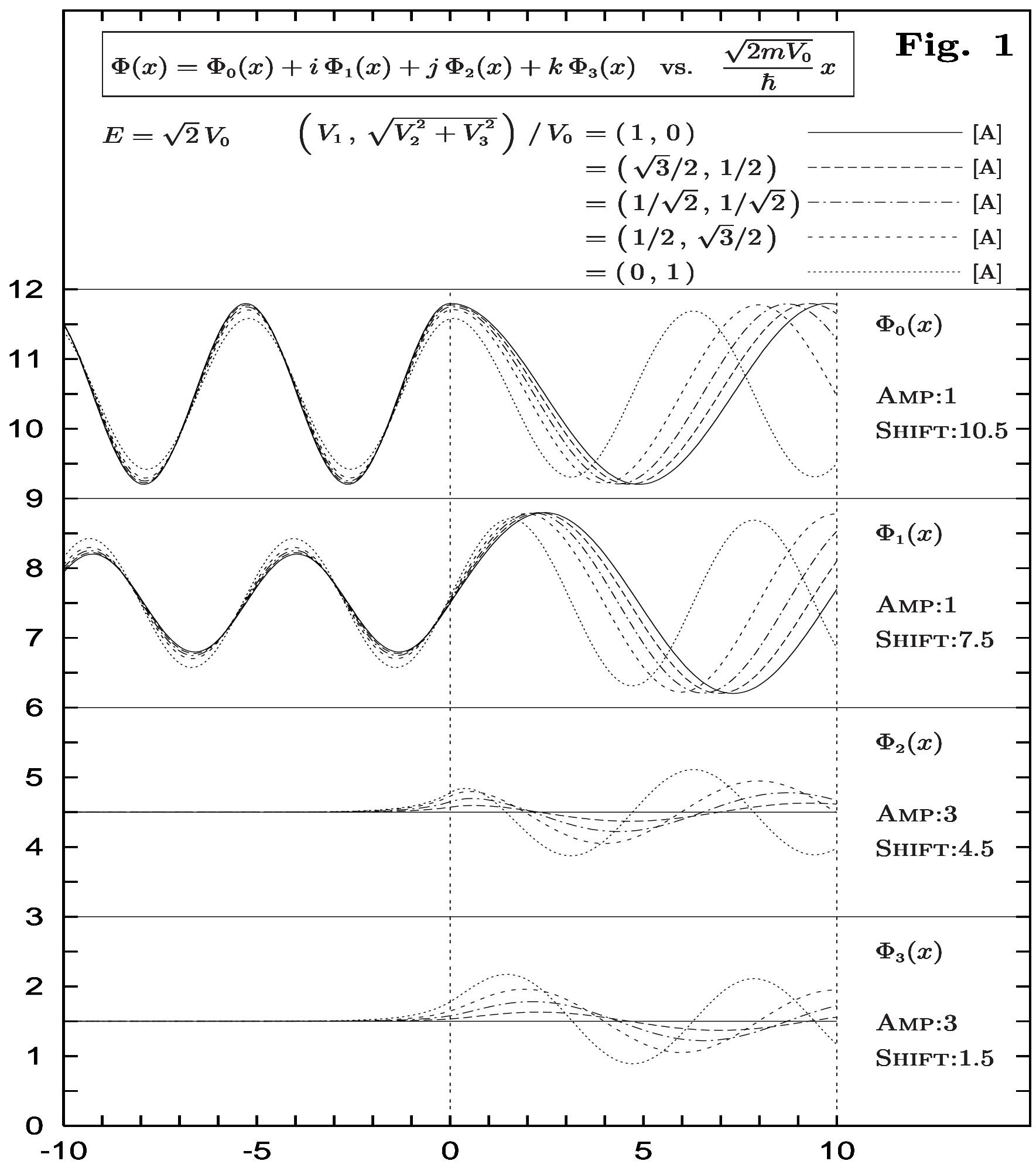

Fig. 1. Space is divided into region I $(x<0)$ and region II $(x>0)$. There is a constat quaternionic potential $\left(i V_{1}+j V_{2}+k V_{3}\right)$ in region II whereas in region I there is no potential. The space dependence of the quaternionic stationary wave function $\Phi(x)$ is plotted for the energy zone $\mathrm{A}\left(E>V_{0}=\sqrt{V_{1}^{2}+V_{2}^{2}+V_{3}^{2}}\right)$ and for different 


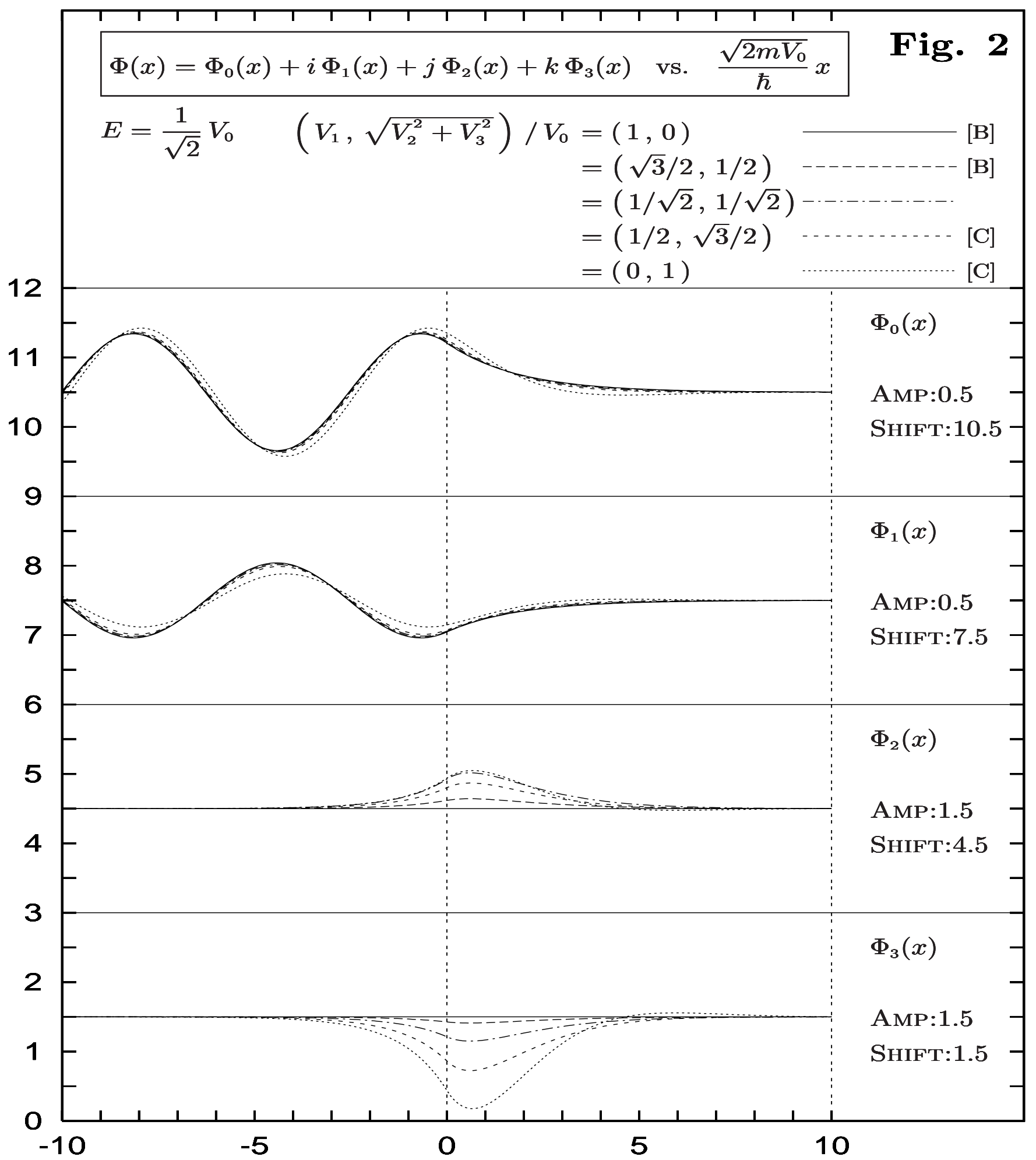

Fig. 2. The space dependence of the quaternionic stationary wave function $\Phi(x)$ is plotted for the energy zone $\mathrm{B}\left(\sqrt{V_{2}^{2}+V_{3}^{2}}<E<V_{0}\right)$ and $\mathrm{C}\left(E<\sqrt{V_{2}^{2}+V_{3}^{2}}\right)$, and for different complex/pure quaternionic potential ratios. The plots for the complex part of $\Phi(x)$ exhibit an evanescent behavior in region II. The pure quaternionic 